\title{
Towards accessible nanophotonics: multimode interferometer on strip-loaded slot waveguide
}

\author{
M. Roussey ${ }^{*}, R$. Rao $^{l}$, and S. Pélisset ${ }^{l}$ \\ ${ }^{1}$ University of Eastern Finland, Institute of Photonics, Joensuu, FI-80100, Finland
}

\begin{abstract}
Nano- and micro-photonics are the key-enabling tools for future integrated components and circuitry operating at low power and high speed. By using a strip-loaded platform, we show how we can dramatically reduce the complexity, in terms of fabrication and tolerances, of the most advanced devices. Different configurations of multimode interference devices are presented. We show the design, fabrication, and optical characterization of these components.
\end{abstract}

\section{Introduction}

A strip-loaded waveguide consists of a high refractive index thin film, in which light is confined laterally by a strip patterned on to of it [1]. This structure offers a high level of degree of freedom in terms of materials, features, and fabrication constraints. The guiding layer can therefore be of any kind of material since it is not patterned and does not need to be processed after deposition. Lithium niobate, glasses, titanium dioxide strip loaded waveguides have been demonstrated successfully in the past years. The loading strip must have a refractive index lower than the effective index of the guided slab mode in order to avoid leaking of light in it. Polymer strips are ideal due to their relatively low refractive index and easiness to pattern. We have recently demonstrated that such a strip-loaded waveguide can be easily combined with a horizontal slot waveguide [2] in order to confine at the nanoscale the electric field in a low refractive index layer sandwiched between two high refractive index films $[3,4]$.

A strip-loaded waveguide is a low refractive index contrast waveguide. This implies larger features, i.e., easier fabrication, and a higher tolerance to imperfections due to fabrication.

\section{Results and discussion}

In this work, we present a multimode interference (MMI) device on a strip-loaded horizontal slot waveguide (SLSW) platform. The waveguide is composed of two layers of titanium dioxide $(\mathrm{nTiO} 2=2.27$ at $\lambda=1550 \mathrm{~nm})$ of thickness $200 \mathrm{~nm}$ separated by an $80 \mathrm{~nm}$ layer of silica $(\mathrm{nSiO} 2=1.444)$. The loading strip is made of $\mathrm{AZ} \mathrm{nLOF}$, an electron beam resist. Figure 1a) depicts, in a top view, the geometrical parameters of the structure. The waveguides width is set to $1.2 \mu \mathrm{m}$ to achieve single mode condition. However, to smooth the coupling to the free propagation region, the waveguides are tapered to $5 \mu \mathrm{m}$ width. Several devices have been realized $(1 \times 2,1 \times 3,1 \times 4$, and $2 \times 2$ ). Only the $1 \times 2$ is presented in Figure 1 .

Finite Different Time Domain (FDTD) simulations have been used to calculate both the modes and the behavior of the field inside the devices. Because of the low index contrast of the waveguide, we were able to use the effective index approximation for the quasi-TM mode (fundamental slot mode) allowing a more refined mesh in a two-dimensional simulation. The field intensity is presented in Figure 1b). We can clearly see the interference pattern in the free propagation region leading to the splitting at the output.

The devices were fabricated by atomic layer deposition for the slot waveguide and electron beam lithography for the patterning of the strip. Figure 1c) shows images obtained with a microscope at $\times 20$ and $\times 50$ magnifications. We can remark that some stitching between the waveguides and the free propagation region appears. This is due to the fact that two different doses have been used for the patterning in order to obtain the best quality in terms of corner of the MMI and fastest writing speed. Using tapers, allows reducing the effect of such a defect in the patterning.

The devices were characterized using tapered lens fibers to inject light and a x20 microscope objective and an infrared camera to observe the output modes. Figure 1d) shows this result proving the correct operation of the device despite the defects occurring during the fabrication.

* Corresponding author: matthieu.roussey@uef.fi 

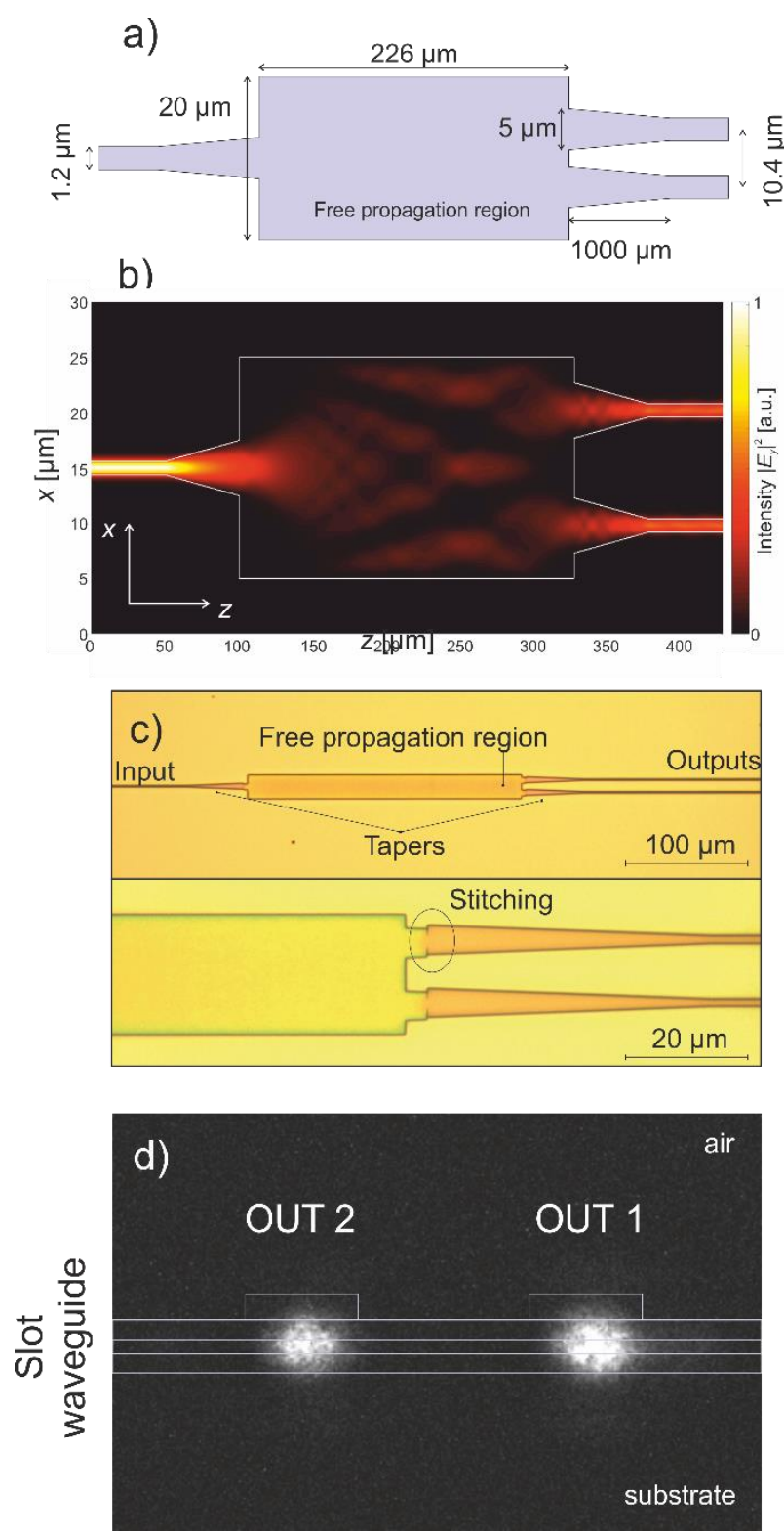

Fig. 1. a) Sketch and geometrical parameters of a 1x2 MMI device on SLSW platform. b) FDTD simulation of the device. The intensity distribution viewed from top is displayed. c) Micrographs of the fabricated device (top: 20x, bottom: 50x). d) Picture taken from the output of the device when illuminated under TM polarization at $\lambda=1550 \mathrm{~nm}$.

\section{Conclusions}

Using the SLSW platform to create an MMI device proves that we can produce standard key building blocks of integrated photonics using reliable fabrication techniques. It is to be noted that such devices can also be fabricated by mass-production methods instead of electron beam lithography, such as nano-imprinting as we demonstrated in [5].

The work is part of the Academy of Finland Flagship Programme, Photonics Research and Innovation (PREIN), decision 320166.

\section{References}

1. H. Furuta, H. Noda, A. Ihaya, "Novel optical waveguide for integrated optics," Appl. Opt. 13, 322326 (1974).

2. V.R. Almeida, Q. Xu, C.A. Barrios, M. Lipson, "Guiding and confining light in void nanostructure," Opt. Lett. 29, 1209-1211 (2004).

3. M. Roussey, L. Ahmadi, S. Pélisset, M. Häyrinen, A. Bera, V. Kontturi, J. Laukkanen, I. Vartiainen, S. Honkanen, M. Kuittinen, "Strip-loaded horizontal slot waveguide," Opt. Lett. 42, 211-214 (2017).

4. S. Pélisset, J. Laukkanen, M. Kuittinen, S. Honkanen, M. Roussey, "Modal properties of a strip-loaded horizontal slot waveguide," J. Eur. Opt. Soc. - Rapid Publ. 13:37 (2017).

5. L. Ahmadi, V. Kontturi, J. Laukkanen, S. Honkanen, M. Kuittinen, M. Roussey, "Strip-loaded waveguide on titanium dioxide thin films by nanoimprint replication", Optics Letters, 42(3) 527-530 (2017). 